\title{
EULAR Points to Consider for the use of imaging to guide interventional procedures in patients with rheumatic and musculoskeletal diseases (RMDs)
}

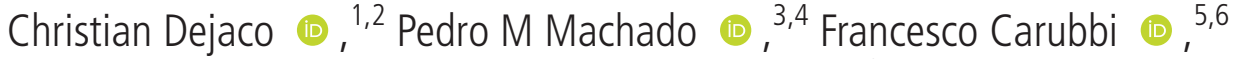 \\ Philipp Bosch (10 , ${ }^{1}$ Lene Terslev, ${ }^{7}$ Giorgio Tamborrini, ${ }^{8}$ Luca Maria Sconfienza, ${ }^{9,10}$ \\ Carlo Alberto Scirè (ㄷ) ,11,12 Sebastian Ruetten, ${ }^{13}$ Jef van Rompay, ${ }^{14}$ Fabian Proft (), ${ }^{15}$ \\ Costantino Pitzalis (1), ${ }^{16}$ Marina Obradov ${ }_{1}^{17}$ Rikke Helene Moe 다, ${ }^{18}$ \\ Vasco V Mascarenhas, ${ }^{19,20}$ Clara Malattia, ${ }^{21,22}$ Andrea Sabine Klauser ${ }^{23}{ }^{2}$ Alison Kent, ${ }^{24}$ \\ Lennart Jans, ${ }^{25}$ Wolfgang Hartung (1), ${ }^{26}$ Hilde Berner Hammer (1), ${ }^{27,28}$ \\ Christina Duftner (10), ${ }^{29}$ Peter V Balint, ${ }^{30}$ Alessia Alunno (i) , ${ }^{5}$ Xenofon Baraliakos (i) ${ }^{31}$
}

Handling editor Josef $S$ Smolen

- Additional supplemental material is published online only. To view, please visit the journal online (http://dx.doi. org/10.1136/annrheumdis2021-221261).

For numbered affiliations see end of article.

\section{Correspondence to}

Dr Christian Dejaco, Department of Rheumatology, Medical University of Graz, Graz 8036, Steiermark, Austria;

christian.dejaco@gmx.net

The results of this study have been presented at the EULAR conference 2021.

Received 28 July 2021 Accepted 23 November 2021

Check for updates

(c) Author(s) (or their employer(s)) 2021. No commercial re-use. See rights and permissions. Published by BMJ.

To cite: Dejaco C

Machado PM, Carubbi F, et al Ann Rheum Dis Epub ahead of print: [please include Day Month Year]. doi:10.1136/ annrheumdis-2021-221261

\section{ABSTRACT}

Objectives To develop evidence-based Points to Consider (PtC) for the use of imaging modalities to guide interventional procedures in patients with rheumatic and musculoskeletal diseases (RMDs).

Methods European Alliance of Associations for Rheumatology (EULAR) standardised operating procedures were followed. A systematic literature review was conducted to retrieve data on the role of imaging modalities including ultrasound (US), fluoroscopy, MRI, CT and fusion imaging to guide interventional procedures. Based on evidence and expert opinion, the task force (25 participants consisting of physicians, healthcare professionals and patients from 11 countries) developed PtC, with consensus obtained through voting. The final level of agreement was provided anonymously. Results A total of three overarching principles and six specific PtC were formulated. The task force recommends preference of imaging over palpation to guide targeted interventional procedures at peripheral joints, periarticular musculoskeletal structures, nerves and the spine. While US is the favoured imaging technique for peripheral joints and nerves, the choice of the imaging method for the spine and sacroiliac joints has to be individualised according to the target, procedure, expertise, availability and radiation exposure. All imaging guided interventions should be performed by a trained specialist using appropriate operational procedures, settings and assistance by technical personnel. Conclusion These are the first EULAR PtC to provide guidance on the role of imaging to guide interventional procedures in patients with RMDs.

\section{INTRODUCTION}

Interventional procedures such as fluid aspiration, injections and biopsies are conducted for diagnostic and therapeutic purposes in patients with different rheumatic and musculoskeletal diseases (RMDs). ${ }^{1}$ Real-time visualisation of the needle or instruments by ultrasound (US), CT, MRI or fluoroscopy has the potential to ensure reliable placement of the needle tip/instrument in the respective anatomical area and to monitor the success of various interventions such as synovial fluid aspiration, drug injection
Key messages

What is already known about this subject?

- Imaging is increasingly used to guide interventional procedures in patients with rheumatic and musculoskeletal diseases (RMDs).

- Imaging guided procedures require additional preparation and training as compared with palpation guided interventions.

- Uncertainty persist among clinicians on which imaging technique should be used to optimally guide interventional procedures.

What does this study add?

- These are the first European Alliance of Associations for Rheumatology endorsed Points to Consider (PtC) for the use of imaging to guide interventional procedures in patients with RMDs.

How might this impact on clinical practice or future developments?

- These PtC give advice to clinicians in which clinical situation, for which intervention, and in which anatomical area imaging should be used to guide interventional procedures.

- The research agenda highlights the gaps in evidence and areas of future studies.

and/or tissue biopsy. ${ }^{2-6}$ Imaging guided procedures, however, are also more resource consuming than conventional palpation guided interventions, require additional preparation and training, and there are some studies suggesting that the outcomes of palpation and imaging guided interventions are not meaningfully different. ${ }^{78}$ Clinicians are therefore still uncertain in which clinical situation, for which intervention, and in which anatomical area imaging should be used to guide interventional procedures. Advice is also needed for the setting (eg, sterility of the room, assistance by nurses) and procedural techniques (eg, direct vs indirect aspiration/injection technique). 
Box 1 Preparations to conduct interventions with direct imaging guidance in situations of low (L) and high risk of infection (H)

- Disinfection of the hands (L) or handwashing and disinfection (H).

- Gloves (L), alternatively sterile gloves (H).

- Sterile preparation of equipment $(\mathrm{L}, \mathrm{H})$.

- Disinfection of the injection site (L, H).

- For US guided interventions.

- Maintenance of at least $0.5 \mathrm{~cm}$ between the probe/gel ${ }^{*}$ and the needle (L) or

- Extensive disinfection of the probe and use of antiseptic instead of gel (L).

- Sterile vinyl foil cover for the US probe and sterile gel (H).

- Face maskst and cap (H).

- Sterilised wraps with opening to expose the applicable site only $(\mathrm{H})$.

*Use of sterile gel for ultrasound guided interventions is recommended by some national authorities.

tObligatory for patients and healthcare provider in several countries during the COVID-19 pandemic.

$L$, low risk of infection; $\mathrm{H}$, high risk of infection; US, ultrasound.

The broad objective of this project is to provide European Alliance of Associations for Rheumatology (EULAR) endorsed, evidence-based Points to Consider $(\mathrm{PtC})$ for the use of imaging to guide interventional procedures in patients with RMDs.

\section{METHODS}

After approval by the EULAR Council, the convenors (CDejaco and $\mathrm{XB}$ ) and the methodologist (PMM) led a task force guided by the 2014 updated EULAR standardised operating procedures (SOPs). ${ }^{9}$ The 25 task force members consisted of rheumatologists, radiologists (all were members of the European Society of Musculoskeletal Radiology), orthopaedic surgeons, patient representatives, methodologists, a healthcare professional and two EMerging EUlar NETwork representatives from 11 countries. All members disclosed their potential conflicts of interest before the start of the process. A hygienist (which is a specialist committed to the prevention of intrahospital infections, including the prevention of surgical site infections), external to the task force, was consulted to discuss and advise the task force regarding the proposals on preparations to conduct interventions with direct imaging guidance detailed in box 1 . One faceto-face and two virtual task force meetings took place, as well as interim email based feedback on the draft PtC. The second meeting was originally scheduled as a face-to-face meeting, but was then transformed into a virtual event due to the restrictions imposed by the COVID-19 pandemic. ${ }^{9}$ Since another face-toface meeting was recommended by EULAR SOPs in order to discuss and vote on the final $\mathrm{PtC}$, a third meeting was scheduled but had ultimately to be transformed again into a webinar due to COVID-19.

At the first meeting (face-to-face), the task force agreed on three broad research questions: (1) What is the value of imaging methods (US, CT, MRI, fluoroscopy/X-ray, fusion imaging) to guide interventional procedures in patients with RMDs, (2) what is the value of different imaging settings and technical standards and (3) what is the value of different procedural techniques for imaging guided interventions.
A single systematic literature review (SLR) was conducted by two fellows (PB and FC) under the guidance of the methodologist (PMM). The convenors, together with the methodologist and fellows translated the research questions in the PICO (Population, Intervention, Comparator, Outcome) format (see online supplemental table 1). ${ }^{10}$ The search strings were developed by an experienced librarian (LF) and applied to MEDLINE, EMBASE, the Cochrane Library and Epistemonikos databases (through 10/21). Prospective and retrospective full research articles, short reports and letters including original (patient) data, published in English and comparing different (imaging) techniques, different settings and procedural protocols to guide interventions in patients with RMDs were retrieved. Risk of bias (RoB) was assessed using the Cochrane RoB tool for randomised trials version 2 (ROB2), the RoB tool for Non-randomized Studies of Interventions and the Appraisal Tool for Cross-Sectional Studies. ${ }^{11-13}$ The evidence summarised in the SLR was presented during the second and third task force meetings. Data were summarised in the form of tables including the RoB assessment. The SLR is published separately; however, it forms an integral and inseparable part of the present $\mathrm{PtC}$ manuscript and should be read as such.

At the second meeting (virtual), the task force formulated the $\mathrm{PtC}$ based on the evidence and expert opinion in a process of discussion and consensus. Subsequently, the draft PtC underwent structured written feedback from the task force members. At the third meeting (virtual), the PtC were refined based on the updated evidence (ie, articles published between second and third task force meeting) and feedback received, followed by voting on the PtC. Consensus was accepted if $>75 \%$ of the members voted in favour of the $\mathrm{PtC}$ at the first round, $\geq 67 \%$ at the second round and at a third round $>50 \%$ was accepted. ${ }^{14}$ The Oxford centre for evidence based medicine 2011 levels of evidence (LoE) derived from the SLR were added to each PtC. ${ }^{15}$

Subsequently, each task force member anonymously indicated the level of agreement via Survey Monkey (LoA, 0-10 numeric rating scale ranging from $0=$ 'completely disagree' to $10=$ 'completely agree'). The mean and SD of the LoA, as well as the percentage of task force members with an agreement $\geq 8$ are presented.

Based on the gaps in the evidence and controversial points, a research agenda was formulated. The manuscript was reviewed by the EULAR Council and a revised version was finally approved by all task force members and the EULAR Council.

\section{RESULTS}

\section{General aspects}

These $\mathrm{PtC}$ are intended to advise qualified (physician and nonphysician) healthcare professionals including rheumatologists, paediatricians, orthopaedic surgeons, neurosurgeons, radiologists, specialists in physical medicine and rehabilitation or sports medicine, general practitioners, anaesthesiologists and physical therapists on the use of imaging modalities to guide interventional procedures in patients with RMDs.

These PtC are not intended to cover all aspects of interventional procedures; we explicitly excluded interventions with the purpose of local or regional anaesthesia before surgery, interventions concerning tumours, vessels or glands as well as arthroplasty and vertebroplasty.

The task force defined 'targeted' interventions as procedures requiring a high level of precision to reach a specific anatomical area such as injection of small ganglia, cysts or tenosynovitis, aspiration of small amounts of fluid or synovial biopsy. 
Table 1 EULAR Points to Consider (PtC) for the use of imaging to guide interventional procedures in patients with rheumatic and musculoskeletal diseases (RMD)

\begin{tabular}{|c|c|c|}
\hline Overarching principles & LoE & LoA \\
\hline $\begin{array}{l}\text { A. The imaging technique should be optimised according to the procedure and the anatomical site taking into account potential side effects, radiation } \\
\text { exposure, availability, expertise and costs. }\end{array}$ & n.a. & $\begin{array}{l}10.0(0.2) \\
100 \%>8\end{array}$ \\
\hline B. Imaging guided interventional procedures should be conducted under adequate aseptic conditions (as detailed in box 1). & n.a. & $\begin{array}{l}10.0(0.2) \\
100 \%>8\end{array}$ \\
\hline C. Complex imaging guided interventional procedures should be conducted with adequate assistance by technical personnel. & n.a. & $\begin{array}{l}9.5(1.7) \\
91.7 \%>8\end{array}$ \\
\hline \multicolumn{3}{|l|}{ Specific Points to Consider } \\
\hline $\begin{array}{l}\text { 1. Imaging should be preferred over palpation to guide targeted* interventional procedures at peripheral joints and periarticular structures in patients } \\
\text { with RMDs. }\end{array}$ & $3 \dagger$ & $\begin{array}{l}9.7(0.5) \\
100 \%>8\end{array}$ \\
\hline 2. Ultrasound should be used as the first imaging modality for interventional procedures at peripheral joints. Fluoroscopy may be used as an alternative. & $3 \dagger$ & $\begin{array}{l}9.1(2.1) \\
95.8 \%>8\end{array}$ \\
\hline $\begin{array}{l}\text { 3. Imaging should be preferred over palpation to guide targeted* injections at structures encompassing peripheral nerves. Ultrasound should be the } \\
\text { preferred imaging modality. }\end{array}$ & $3 \dagger$ & $\begin{array}{l}9.9(0.3) \\
100 \%>8\end{array}$ \\
\hline 4. Imaging should be used to guide targeted ${ }^{*}$ injections at the spine. & 5 & $\begin{array}{l}9.9(0.3) \\
100 \%>8\end{array}$ \\
\hline 5. Imaging should be preferred over palpation for targeted* injections of the sacroiliac joint(s). & $3+$ & $\begin{array}{l}9.9(0.3) \\
100 \%>8\end{array}$ \\
\hline $\begin{array}{l}\text { 6. Healthcare professionals performing imaging guided interventional procedures must have adequate skills in the respective imaging technique and the } \\
\text { interventional procedure. }\end{array}$ & 5 & $\begin{array}{l}8.9(2.9) \\
87.5 \%>8\end{array}$ \\
\hline
\end{tabular}

Numbers in column 'LoA' indicate the mean and SD (in parenthesis) of the LoA (range $0-10$ with $0={ }^{\prime}$ completely disagree' to $10=$ 'completely agree'), as well as the percentage of task force members with an agreement $\geq 8$.

*Targeted interventions are defined as procedures requiring a high level of precision to reach a specific anatomical area such as injection of small ganglia, cysts or tenosynovitis, including aspiration of small amounts of fluid or synovial biopsy.

tLevels of evidence were downgraded (from level 2 to level 3) because of bias related to randomisation, outcome assessment (trials and non-randomised studies), the population of interest (cross-sectional studies) and inadequate adjustment of potential confounders.

EULAR, European Alliance of Associations for Rheumatology; LoA, level of agreement; LoE, level of evidence; n.a., not applicable; RMDs, rheumatic and musculoskeletal diseases.

The population of interest is patients with RMDs (degenerative, inflammatory or autoimmune) including patients with painful joints, tendons, entheses and/or muscles, as well as neuropathic pain or discomfort.

These PtC may also inform patients participating in shareddecision making and healthcare provider organisations arranging care for patients with RMD.

A total of three overarching principles and six specific $\mathrm{PtC}$ have been formulated. They are summarised in table 1 (including the LoE and LoA) and are discussed in detail below.

\section{Overarching principles}

These refer to principles of a generic nature. They are not necessarily based on specific LoE but reflect issues of good clinical practice and the task force considered them as a framework for the subsequent, specific PtC.

A. The imaging technique should be optimised according to the procedure and the anatomical site taking into account potential side effects, radiation exposure, availability, expertise and costs.

The term 'technique' refers not only to the choice of the imaging device, but also to the technical procedure such as direct visualisation of the needle during the intervention as compared with indirect imaging guidance where the exact position of the target is marked first using imaging followed by blind intervention, in or out of plane needle guidance and so on, and the materials used such as different types of needles or other devices. Imaging may also support the decision of whether an intervention will be conducted by palpation or imaging guidance. A joint filled with synovial proliferation for example, might be markedly swollen but contain a small amount of fluid only. Aspiration guided by palpation might not be successful in such a situation, while imaging may help to reach the target easily. Another, less intuitive application of imaging is to confirm whether a target has been reached by an intervention guided by palpation. Evidence from the literature is absent for the majority of these aspects and the material/equipment available might differ between countries and hospitals/practices. High level of expertise for a given procedure was considered more relevant than developing a standard protocol for every possible situation, therefore, the task force made a specific PtC on skills and training below. In addition, radiation exposure should be balanced against expected accuracy and procedural safety of the intervention, and judgement may be different when the intervention is performed in young adults or children as compared with elderly people.

A related aspect is the relevance of in-of-target versus out-oftarget interventions. For some indications such as injection of trigger finger, clinical studies reported no difference for whether an injection was inside or outside flexor tendon sheaths in terms of safety and clinical efficacy. ${ }^{16}{ }^{17}$ In contrast, studies on joint interventions reported higher levels of pain in case glucocorticoid injections were extra-articular as compared with intraarticular. ${ }^{18} 19$ For epidural injection of the spine or tissue biopsy, in-target placement of the needle is mandatory in order to avoid nerve damage or to obtain a representative biopsy sample, respectively. An overview of clinical studies retrieved by the SLR comparing different procedural protocols for imaging guided interventions is depicted in table 2. Details of the studies cited are summarised in the SLR accompanying these PtC. ${ }^{10}$

B. Imaging guided interventional procedures should be conducted under adequate aseptic conditions.

While every procedure penetrating the skin of a patient must be aseptic, the level of sterility may vary. The type of intervention (eg, injection vs biopsy or direct vs indirect imaging guidance), the anatomical site (eg, enthesis vs spine) and the immunocompetency of the patient are some of the factors that may influence how 'aseptic' the setting should be.

Studies comparing different measures to guarantee the sterility of imaging guided interventional procedures are absent, and 
Table 2 Overview of studies identified by the systematic literature review investigating different procedural protocols for imaging guided interventions in patients with rheumatic and musculoskeletal disease

\begin{tabular}{|c|c|c|}
\hline Intervention & Comparator & Results for intervention \\
\hline Intra-articular injections in sacroiliitis and ACJ arthritis ${ }^{81819}$ & Periarticular & Superior for short-term, ${ }^{19}$ and long-term pain ${ }^{18}$ \\
\hline Shoulder joint injections in adhesive capsulitis ${ }^{4647}$ & SASD bursa & $\begin{array}{l}\text { Superior for short-term, }{ }^{47} \text { and long-term pain. }{ }^{46} 47 \text { Mixed results for } \\
\text { efficacy }\end{array}$ \\
\hline Subscapularis muscle injection in scapular pain ${ }^{48}$ & Scapulothoracic bursa & No difference in safety and efficacy \\
\hline Medial access for knee injections in $\mathrm{OA}^{4950}$ & Midlateral/superolateral access & No difference in safety and accuracy \\
\hline US in-plane injection in knee $\mathrm{OA}^{51}$ & US out-of-plane & No difference in accuracy, adverse events or procedural time \\
\hline Bone biopsy in suspected osteomyelitis ${ }^{52}$ & Paravertebral soft tissue & No difference in tissue acquisition \\
\hline Intra-tendon sheath injection in trigger fingers ${ }^{5354}$ & Extra tendon sheath & No difference in safety and efficacy \\
\hline Intra-epineurium injections in $\mathrm{CTS}^{29}$ & Extra-epineurium & Superior for symptom severity and efficacy \\
\hline Ulnar access for injection in $\mathrm{CTS}^{55} 56$ & Midline/radial access & Inferior for long-term pain reduction compared with radial access ${ }^{55}$ \\
\hline Injection above the median nerve in $\mathrm{CTS}^{57}$ & Injection under the median nerve & No difference in safety and efficacy \\
\hline
\end{tabular}

ACJ, acromioclavicular joint; CTS, carpal tunnel syndrome; OA, osteoarthritis; SASD, subacromial/subdeltoid; US, ultrasound.

most of the studies retrieved by the SLR were relatively vague in the description of what preparations were made..$^{10}$ Based on expert opinion, and considering current clinical practice, the task force proposed preparations to conduct interventions with direct imaging guidance under aseptic conditions in relation to the presumed risk of infection (box 1). Preparation of procedures with indirect imaging guidance (ie, conduction of imaging first followed by a blind intervention) are identical to palpation guided interventions described elsewhere. ${ }^{1}$ The suggestions in box 1 are not intended to cover every clinical situation nor to reflect all national guidelines. Some authorities for example, recommend using sterile gel for US guided interventions which is not current practice in every EULAR country. ${ }^{20}{ }^{21}$ In a severely immunocompromised patient undergoing highly invasive interventions (eg, tissue biopsy at the spine) even more intensive preparations than those listed in box 1 (such as using an operation theatre, surgical aseptic hand washing and wearing surgical gowns) may be required to minimise the risk of infection. Likewise, face masks are obligatory during the COVID-19 pandemic in many countries for patients, physicians and healthcare professionals along with a negative SARS-CoV-2 test for patients, however, it is not clear, whether face masks reduce the risk of infection in simple imaging guided interventions such as joint injections, once the pandemic is over.

C. Complex imaging guided interventional procedures should be conducted with adequate assistance by technical personnel.

The task force agreed that complex imaging guided procedures such as synovial tissue biopsies should be supported by technical personnel. Simple interventions such as US guided intra-articular injections could, at least in theory, be managed without assistance even though the experts were of the opinion that every imaging guided intervention benefit from assistance, particularly to maintain sterility of the setting and to ensure a high accuracy of the procedure. Technical personnel are also required to prepare equipment and drugs, to assist the procedure and to help monitoring of patients' clinical status during and after the procedure, when needed. Literature is scarce about the possible benefit of technical assistance for the prevention of adverse events as well as for cost-effectiveness; these issues should be clarified by future studies.

\section{Specific PtC}

Point to Consider 1

Imaging should be preferred over palpation to guide targeted interventional procedures at peripheral joints and periarticular structures in patients with RMDs.
The task force recognised that not all interventions at peripheral joints and periarticular structures (which include tendons, ligaments, entheses, pulleys and bursae) require imaging guidance, that imaging is not available in every setting and/or that professionals conducting interventions may not have sufficient expertise with imaging guidance. Synovial fluid aspiration of an extensively swollen knee, non-targeted injection of a metacarpophalangeal joint in a patient with rheumatoid arthritis, injection of the subacromial bursae in a patient with rotator cuff disease, injection of a trigger finger or enthesitis at lateral epicondyle might well be guided by palpation. In contrast, targeted interventions should be conducted under imaging guidance in order to guarantee a high accuracy of the procedure. The absence of immediate access to imaging, however, should not delay an urgent diagnostic procedure such as arthrocentesis in case of suspected septic arthritis.

Evidence from clinical studies indicate a better accuracy (including correct needle placement and superiority in tissue and fluid acquisition) and safety (less procedural and postprocedural pain and discomfort) for imaging than for palpation guided interventions whereas data regarding short-term and long-term efficacy are contrasting. ${ }^{10}$ The most important limitation of these studies, however, is that they did not detangle easy (eg, subcapsular space of a highly swollen joint) from difficult to reach targets (eg, small ganglion compressing a peripheral nerve). Accordingly, the task force had to extrapolate the evidence to conclude that imaging should be preferred when a high level of precision is needed in order to reach a specific anatomical area.

Studies on costs of imaging guided interventions at peripheral joints are available only for the USA reporting large differences of costs depending on the setting and reimbursement policies of individual insurance companies. ${ }^{22-24}$ Whether imaging guided interventions are cost-effective in the USA and EULAR countries (eg, by preventing secondary direct and indirect costs due to higher efficacy and/or lower rate of complications) is unclear so far. This aspect has been added to the research agenda.

\section{Point to Consider 2}

Ultrasound should be used as the first imaging modality for interventional procedures at peripheral joints. Fluoroscopy may be used as an alternative.

The majority of studies at peripheral joints were available for US and fluoroscopy with comparable results concerning efficacy and accuracy. ${ }^{10}$ While fluoroscopy is still widely used in clinical practice, ${ }^{25}$ the task force agreed that US should be preferred over fluoroscopy if both techniques were available with similar 
expertise, because of the absence of radiation, the better visualisation of soft tissue and the lower resource consumption by the former, as well as the fact that US can be used as part of everyday clinical practice. $^{24} 26$ The European Union directive 2013/59/ EURATOM states that if a non-radiating imaging modality is available, it should be invariably used and preferred over a modality which uses ionising radiations. ${ }^{27}$ Fluoroscopy is a valid alternative, particularly if US is not available, for joint aspiration and intra-articular injections. ${ }^{28}$

Other imaging modalities to guide interventional procedures of peripheral joints such as CT, MRI or fusion imaging are still a matter of research.

\section{Point to Consider 3}

Imaging should be preferred over palpation to guide targeted injections at structures encompassing peripheral nerves. Ultrasound should be the preferred imaging modality.

The task force emphasised that imaging is particularly helpful when a specific target, for example, a cyst or ganglion compressing a peripheral nerve, should be injected. One study reported a higher efficacy of intraepineural than extraepineural injection of the median nerve in patients with carpal tunnel syndrome for symptom improvement as well as for reduction of nerve swelling. ${ }^{29}$ It is almost impossible to safely reach such a small anatomical place without imaging, even though a comparison between imaging and palpation guidance for this intervention is still missing.

The highest number of studies, most of them with low quality, were available for the comparison between US and palpation guided injections at the carpal tunnel. ${ }^{10}$ Some of them reported more adverse events in patients undergoing palpation guided injections (eg, hand weakness, finger numbness, skin discolouration or subcutaneous fat atrophy) ${ }^{30} 31$ whereas others found no difference in terms of safety and efficacy. ${ }^{10}$ The task force members recognised that most studies might have been underpowered to detect rare adverse events such as accidental nerve puncture or injury of the persistent median artery, particularly in patients with anatomical variants of the median nerve. Based on clinical experience, such adverse events can easily be avoided if imaging is used to guide the injections. A bifid median nerve is the most common anatomical variant occurring in $15 \%-20 \%$ of the population, $11 \%$ have a persistent median artery. ${ }^{32} 33$

Evidence on imaging guided injections at peripheral nerves outside the carpal tunnel is scarce and mainly derives from observational and cadaveric studies, ${ }^{34-39}$ hence, this aspect has been included in the research agenda.

Fluoroscopy is not recommended for this indication because of the absence of data from trials and the fact that nerves cannot be visualised directly with this technique. The value of other imaging methods such as MRI, or CT with/without fusion with US to guide interventions at peripheral nerves still needs to be elucidated.

\section{Point to Consider 4}

Imaging should be used to guide targeted injections at the spine.

It is common clinical practice to use imaging for injections at the spine as demonstrated by a recent survey and according to experience of the task force members. ${ }^{25}$ In clinical practice, the choice of the technique depends on the target (US or fluoroscopy may be adequate for injections at facet joints whereas peri-radicular and epidural injections are mostly guided by CT), disease stage (CT or fusion imaging between CT/US or MRI/ US may be used in cases with advanced degenerative disease or other structural damage at the spine), ${ }^{40} 41$ local expertise and availability. Clinical studies comparing different techniques are virtually absent; a single study compared fluoroscopy with CT to conduct biopsies in case of suspected vertebral osteomyelitis revealing a better performance of the former, given its ability to adjust the needle in a vertical plane. ${ }^{42}$ Facet joint injections are sometimes conducted under clinical guidance, ${ }^{25}$ the percentage of in-target administration of the drug, safety and efficacy of this approach as compared with imaging guidance, however, is probably low (even though direct evidence is missing). MRI is rarely used to guide injections at the spine and there are little data from clinical studies to support its use. ${ }^{10}$

\section{Point to Consider 5}

Imaging should be preferred over palpation for targeted injections of the sacroiliac joint(s).

While injections of the sacroiliac joints are sometimes guided by palpation in clinical practice, the probability to reach the joint space is less than $25 \% .{ }^{43}$ Using imaging to guide injections and other interventions such as synovial tissue biopsy increases the accuracy of the procedure dramatically. One study reported that the joint space was reached in $85 \%$ of cases if US guidance was used, ${ }^{44}$ and others found in-target needle placement in $91 \%$ of fluoroscopy guided injections. ${ }^{28}$ Most efficacy and safety outcomes, however, were similar, independent of whether the injection was intra-articular or periarticular. ${ }^{8} 18$ The follow-up time as well as the power of these studies to detect clinical differences, however, were limited and might thus have underestimated the true benefit of releasing drugs inside rather than outside the joint capsule.

The choice of the most appropriate imaging modality such as US, fluoroscopy, CT and MRI for sacroiliac joint injections is determined by local expertise and availability as well as by considerations of radiation exposure. Fusion imaging between US and CT might be helpful in case bony spurs or other type of joint damage limit the anatomical passage into the joint space. ${ }^{45}$

\section{Point to Consider 6}

Healthcare professionals performing imaging guided interventional procedures must have adequate skills in the respective imaging technique and the interventional procedure.

According to local rules and legal framework, non-physician healthcare professionals may also conduct imaging guided interventions, however, the task force strongly endorses specific training of all professionals performing these procedures. The amount of training depends on the technique and on local training requirements. EULAR has defined competencies in musculoskeletal US, and US guided interventions are part of intermediate and advanced level EULAR US courses, however, the task force considered it beyond the scope of this project to define the specific skills qualifying for imaging guided interventional procedures. Evidence from clinical studies is missing, hence this item has been added to the research agenda.

Based on the discussions and the areas of uncertainty, a research agenda has been proposed, depicted in box 2 .

\section{DISCUSSION}

These are the first EULAR PtC providing up-to-date guidance for the role of imaging to guide interventional procedures in patients with RMDs.

These principles are reflected in both the $\mathrm{PtC}$ and the research agenda, acknowledging also the gaps in evidence that include direct comparisons between different imaging modalities as well 


\section{Box 2 Future research agenda}

- To compare the efficacy and accuracy of interventions guided by US, fluoroscopy, CT, MRI or fusion imaging at peripheral joints, nerves and the spine and for different indications (eg, injections, arthrocentesis or biopsy; inflammatory vs noninflammatory conditions).

- To compare imaging versus palpation guided interventions at different anatomical sites.

- To compare the safety and accuracy of imaging guided interventions conducted with and without technical personnel assisting the procedure.

- To develop and use outcome measures with importance to society including assessment of sick-leave days, costeffectiveness and health resource consumption in studies on interventional procedures.

- To identify and agree on outcomes measuring the success of interventional procedures (eg, amount of fluid aspiration, quality of the samples in case of biopsies, long-term pain reduction in case of injections).

- To study the value of MRI, CT and/or fusion imaging for interventions at peripheral nerves, to study the value of US for interventions at nerves outside the carpal tunnel.

- To study the value of imaging to avoid accidental nerve trauma as compared with palpation guided injections.

- To investigate the effect of specific training programmes on the accuracy of imaging guided interventional procedures and to assess the learning curve of professionals conducting imaging guided interventions.

- To define standard procedural protocols for imaging guided interventions.

- To investigate the effect of different levels of aseptic conditions on the prevalence of infections in imaging guided interventions.

- To evaluate the effect of echo-tip needles and needle visualisation US software for the accuracy of imaging guided interventions.

- To compare different techniques and equipment for imaging guided interventions at different anatomical sites.

US, ultrasound.

as the low amount of data on imaging guided interventions at peripheral nerves (particularly outside the carpal tunnel) and the spine. Besides, outcomes to measure the success of interventions (eg, amount of fluid or quality of samples in case of arthrocentesis or biopsy, respectively, reduction of damage to surrounding structures, long-term pain reduction by injections), are elusive and should be defined by future research.

Where evidence from clinical trials was controversial or absent, PtC were formulated on the basis of current clinical practice and expert opinion. ${ }^{25}$ Good quality studies are now required to answer the numerous questions raised in the research agenda, so that future PtC can be upgraded and based on more solid evidence. The present $\mathrm{PtC}$ nevertheless represent a step forward in the approach to conduct interventional procedures using imaging, complementing recent EULAR recommendations for intra-articular therapies. ${ }^{1}$ We believe that their implementation will improve patient care.

A concern is publication bias assuming that negative studies or studies demonstrating that palpation guided interventions are superior over imaging guidance were probably less frequently published. Another limitation is that the task force was mainly composed of specialists using imaging regularly, even though they also conduct palpation guided interventions routinely. Expert opinion might nevertheless be biased towards a preference of imaging over clinical guidance of interventions.

In summary, we developed three overarching principles and six specific $\mathrm{PtC}$ on the use of imaging for interventional procedures in $\mathrm{RMD}$. These $\mathrm{PtC}$ are supported by evidence along with expert consensus. Unresolved issues and areas of further study have been depicted in the research agenda. We expect that much progress continues taking place in the area of imaging in RMDs, and we will carefully follow developments in the field, assuming that an amendment of these $\mathrm{PtC}$ may be needed within a few years.

\section{Author affiliations}

${ }^{1}$ Department of Rheumatology, Medical University of Graz, Graz, Austria ${ }^{2}$ Department of Rheumatology (ASAA-SABES), Brunico Hospital, Brunico, Italy ${ }^{3}$ Centre for Rheumatology \& Department of Neuromuscular Diseases, University College London, London, UK

${ }^{4}$ National Institute for Health Research (NIHR) University College London Hospitals Biomedical Research Centre, University College London Hospitals NHS Foundation Trust, London, UK

${ }^{5}$ Internal Medicine and Nephrology Unit, University of L'Aquila Department of Clinical Medicine Life Health and Environmental Sciences, L'Aquila, Italy

${ }^{6}$ Department of Medicine, ASL 1 Avezzano-Sulmona-L'Aquila, San Salvatore Hospital, L'Aquila, Italy

${ }_{7}^{7}$ Center for Rheumatology and Spine Diseases, Rigshospitalet, Kobenhavn, Denmark

${ }^{8}$ UZR, Ultraschallzentrum und Institut für Rheumatologie, Basel, Switzerland

${ }^{9}$ Dipartimento di Scienze Biomediche per la Salute, Universita degli Studi di Milano,

Milano, Italy

${ }^{10}$ Diagnostic and Interventional Radiology, IRCCS Istituto Ortopedico Galeazzi,

Milano, Italy

${ }^{11}$ Rheumatology Unit, Department of Medical Sciences, University of Ferrara, Ferrara, Italy

${ }^{12}$ Epidemiology Research Unit, Italian Society of Rheumatology, Milano, Italy

${ }^{13}$ Center for Spine Surgery and Pain Therapy, Center for Orthopedics and

Traumatology, St. Anna Hospital, Herne, Germany

${ }^{14}$ Patient Research Partners, Patient Research, Antwerpen, Belgium

${ }^{15}$ Department of Gastroenterology, Infectiology and Rheumatology, Charite

Universitatsmedizin Berlin Campus Benjamin Franklin, Berlin, Germany

${ }^{16}$ Experimental Medicine and Rheumatology, William Harvey Research Institute,

London, UK

${ }^{17}$ Radiology, Sint Maartenskliniek, Nijmegen, The Netherlands

${ }^{18}$ Division of Rheumatology and Research, Diakonhjemmet Hospital, Oslo, Norway

${ }^{19}$ UIME (Unidade de Imagem Musculo-esquelética), Hospital da Luz Imaging Center, Lisbon, Portugal

${ }^{20}$ Rheumatic Diseases Lab, CEDOC, Universidade Nova de Lisboa, Lisboa, Portugal ${ }^{21}$ UOC Clinica Pediatrica e Reumatologia, Istituto Giannina Gaslini, Genova, Italy

${ }^{22}$ Department of Neurosciences, Rehabilitation, Ophthalmology, Genetic and Maternal Infantile Sciences (DINOGMI), University of Genoa, Genova, Italy

${ }^{23}$ Radiology II, Medical University Innsbruck Department of Radiology, Innsbruck, Austria

${ }^{24}$ Salisbury Hospital NHS Foundation Trust, Salisbury, UK

${ }^{25}$ Radiology, Ghent University Hospital Radiology Department, Gent, Belgium

${ }^{26}$ Department of Rheumatology and Clinical Immunology, Asklepios Medical Center,

Bad Abbach, Germany

${ }^{27}$ Rheumatology, Diakonhjemmet Hospital, Oslo, Norway

${ }^{28}$ Faculty of Medicine, University of Oslo, Oslo, Norway

${ }^{29}$ Department of Internal Medicine, Clinical Division of Internal Medicine II, Medical University Innsbruck, Innsbruck, Austria

${ }^{30} 3$ rd Department of Rheumatology, National Institute for Rheumatology and Physiotherapy, Budapest, Hungary

${ }^{31}$ Rheumatology, Ruhr-University Bochum, Rheumazentrum Ruhrgebiet Herne, Herne, Germany

Twitter Pedro M Machado @pedrommcmachado, Carlo Alberto Scirè @rthritis and Rikke Helene Moe @MoeRikke

Acknowledgements The authors would like to thank Louise Falzon for her work in the development of the literature search strategy, Gaia Piccinni and Elisabeth Kuenzer for their consultation concerning the formulation of box 1 on 'Preparations to conduct interventions with direct imaging guidance'.

Contributors All authors were involved in the discussion and formulation of the Points to Consider. CDejaco wrote the first version of the manuscript. All authors reviewed it and made extensive comments and appropriate changes to it. All authors approved the final version of the manuscript. 
Funding Funding was provided by the European Alliance of Associations for Rheumatology, project number IMG016.

Competing interests CDejaco has received consulting/speaker's fees from Abbvie, Eli Lilly, Janssen, Novartis, Pfizer, Roche, Galapagos and Sanofi, all unrelated to this manuscript. PMM has received consulting/speaker's fees from Abbvie, BMS, Celgene, Eli Lilly, Galapagos, Janssen, MSD, Novartis, Orphazyme, Pfizer, Roche and UCB, all unrelated to this manuscript, and is supported by the National Institute for Health Research (NIHR), University College London Hospitals (UCLH), Biomedical Research Centre (BRC). FC has received consulting/speaker's fees from Abbvie and Celgene, all unrelated to this manuscript. LT has received speakers fee from Novartis, UCB, Roche and Pfizer. GT has received research grants from lqone and received consulting/speaker's fees from Menarini, all unrelated to this manuscript. LMS has received consulting/speaker's fees from Janssen-Cilag, Novartis, Pfizer, Abiogen, Samsung Medison, Esaote, all unrelated to this manuscript. FP has received research grants from Novartis and UCB and received consulting/speaker's fees from Abbvie, AMGEN, BMS, Hexal, Janssen, MSD, Novartis, Pfizer, Roche and UCB, all unrelated to this manuscript. CP has received honoraria and/or research and development grants from: Abbott/AbbVie, Astellas, Astra-Zeneca/Medlmmune, BMS, CelGene, Grunenthal, GSK, Janssen/J\&J, MSD, Pfizer, Sanofi, Roche/Genentech/Chugai, UCB. $\mathrm{HBH}$ has received fees for speaking and/or consulting from AbbVie, BMS, Pfizer, UCB, Roche, MSD and Novartis. WH has received fees for speaking and/or consulting from AbbVie, Alpinion, Canon, Celgene, Chugai, Janssen-Cilag, Pfizer, Roche. CDuftner has received consulting/speaker's fees from Abbvie, BMS, Eli Lilly, Janssen, Novartis, Pfizer, Roche and UCB, all unrelated to this manuscript. PVB has received consulting/ speaker's fees from Abbvie, BMS, Celgene, Celltrion, Eli Lilly, IBSA, Janssen-Cilag, MSD, Novartis, Pfizer, Professional Publishing Hungary, Richter, Roche, Sandoz, Springer Nature, UCB, all unrelated to this manuscript. XB has received consulting/ speaker's fees or grant support from Abbvie, Amgen, BMS, Chugai, Galapagos, Gilead, Hexal, Lilly, MSD, Novartis, Pfizer, Roche, Sandoz, UCB, all unrelated to this manuscript.

\section{Patient consent for publication Not applicable.}

Ethics approval This study does not involve human participants.

Provenance and peer review Not commissioned; externally peer reviewed.

Supplemental material This content has been supplied by the author(s). It has not been vetted by BMJ Publishing Group Limited (BMJ) and may not have been peer-reviewed. Any opinions or recommendations discussed are solely those of the author(s) and are not endorsed by BMJ. BMJ disclaims all liability and responsibility arising from any reliance placed on the content. Where the content includes any translated material, BMJ does not warrant the accuracy and reliability of the translations (including but not limited to local regulations, clinical guidelines, terminology, drug names and drug dosages), and is not responsible for any error and/or omissions arising from translation and adaptation or otherwise.

\section{ORCID iDs}

Christian Dejaco http://orcid.org/0000-0002-0173-0668

Pedro M Machado http://orcid.org/0000-0002-8411-7972

Francesco Carubbi http://orcid.org/0000-0003-1958-5136

Philipp Bosch http://orcid.org/0000-0002-6783-6422

Carlo Alberto Scirè http://orcid.org/0000-0001-7451-0271

Fabian Proft http://orcid.org/0000-0003-4306-033X

Costantino Pitzalis http://orcid.org/0000-0003-1326-5051

Rikke Helene Moe http://orcid.org/0000-0001-7601-5346

Wolfgang Hartung http://orcid.org/0000-0001-9158-3616

Hilde Berner Hammer http://orcid.org/0000-0001-7317-8991

Christina Duftner http://orcid.org/0000-0003-3137-8834

Alessia Alunno http://orcid.org/0000-0003-1105-5640

Xenofon Baraliakos http://orcid.org/0000-0002-9475-9362

\section{REFERENCES}

1 Uson J, Rodriguez-García SC, Castellanos-Moreira R, et al. EULAR recommendations for intra-articular therapies. Ann Rheum Dis 2021;80:1299-305.

2 Günaydin I, Pereira PL, Daikeler T, et al. Magnetic resonance imaging guided corticosteroid injection of the sacroiliac joints in patients with therapy resistant spondyloarthropathy: a pilot study. J Rheumatol 2000;27:424-8.

3 Braun J, Bollow M, Seyrekbasan F. Computed tomography guided corticosteroid injection of the sacroiliac joint in patients with spondyloarthropathy with sacroiliitis: clinical outcome and followup by dynamic magnetic resonance imaging. J Rheumatol 1996:23:659-64.

4 Kelly S, Humby F, Filer A, et al. Ultrasound-Guided synovial biopsy: a safe, welltolerated and reliable technique for obtaining high-quality synovial tissue from both large and small joints in early arthritis patients. Ann Rheum Dis 2015;74:611-7.

5 Klauser AS, Sailer-Hoeck M, Abdellah MMH, et al. Feasibility of ultrasound-guided Sacroiliac joint injections in children presenting with sacroiliitis. Ultraschall Med 2016;37:393-8.
6 Klauser A, De Zordo T, Feuchtner G, et al. Feasibility of ultrasound-guided sacroiliac joint injection considering sonoanatomic landmarks at two different levels in cadavers and patients. Arthritis Rheum 2008;59:1618-24.

7 Nordberg LB, Lillegraven S, Aga A-B, et al. The impact of ultrasound on the use and efficacy of intraarticular glucocorticoid injections in early rheumatoid arthritis. Arthritis Rheumatol 2018:70:1192-9.

8 Hartung W, Ross CJ, Straub R, et al. Ultrasound-Guided sacroiliac joint injection in patients with established sacroiliitis: precise la injection verified by MRI scanning does not predict clinical outcome. Rheumatology 2010;49:1479-82.

9 van der Heijde D, Aletaha D, Carmona L, et al. 2014 update of the EULAR standardised operating procedures for EULAR-endorsed recommendations. Ann Rheum Dis 2015;74:8-13.

10 Bosch P, Carubbi F, Scirè CA, et al. Value of imaging to guide interventional procedures in rheumatic and musculoskeletal diseases: a systematic literature review informing EULAR points to consider. RMD Open 2021;7:e01864.

11 Downes MJ, Brennan ML, Williams HC, et al. Development of a critical appraisal tool to assess the quality of cross-sectional studies (axis). BMJ Open 2016;6:e011458.

12 Sterne JAC, Savović J, Page MJ, et al. Rob 2: a revised tool for assessing risk of bias in randomised trials. BMJ 2019:2:14898

13 Sterne JAC, Hernán MA, Reeves BC, et al. ROBINS-I: a tool for assessing risk of bias in non-randomised studies of interventions. BMJ 2016;355:14919.

14 EUALR. Voting-procedures on EULAR recommendations, 2019. Available: https:// www.eular.org/myUploadData/files/voting_eular_recos_approved_exc_june_2019 web.pdf

15 OCEBM Levels of Evidence Working Group. The Oxford 2011 levels of evidence, 2011. Available: https://www.cebm.ox.ac.uk/resources/levels-of-evidence/ocebm-levels-ofevidence

16 Lee SH, Choi YC, Kang HJ. Comparative study of ultrasonography-guided percutaneous A1 pulley release versus blinded percutaneous A1 pulley release. J Orthop Surg 2018:26:230949901877236.

17 Cecen GS, Gulabi D, Saglam F, et al. Corticosteroid injection for trigger finger: blinded or ultrasound-guided injection? Arch Orthop Trauma Surg 2015;135:125-31.

18 Althoff CE, Bollow M, Feist E, et al. Ct-Guided corticosteroid injection of the sacroiliac joints: quality assurance and standardized prospective evaluation of long-term effectiveness over six months. Clin Rheumatol 2015:34:1079-84.

19 Sabeti-Aschraf M, Stotter C, Thaler C, et al. Intra-Articular versus periarticular acromioclavicular joint injection: a multicenter, prospective, randomized, controlled trial. Arthroscopy 2013;29:1903-10.

20 Ministero della Salute. Raccomandazioni per l'uso sicuro dei gel ecografici, 2021. Available: https://portale.fnomceo.it/wp-content/uploads/2021/05/COMUNICAZIONEN-114-2021.pdf

21 Public Health England. Good infection prevention practice: using ultrasound gel, 2021. Available: https://www.gov.uk/government/publications/ultrasound-gel-goodinfection-prevention-practice/good-infection-prevention-practice-using-ultrasoundgel

22 Mitchell WG, Kettwich SC, Sibbitt WL, et al. Outcomes and cost-effectiveness of ultrasound-guided injection of the trochanteric bursa. Rheumatol Int 2018:38:393-401.

23 Sibbitt WL, Band PA, Kettwich LG, et al. A randomized controlled trial evaluating the cost-effectiveness of sonographic guidance for intra-articular injection of the osteoarthritic knee. J Clin Rheumatol 2011;17:409-15.

24 Henne $\mathrm{M}$, Centurion A, Zeini IM, et al. Trends in utilization of image guidance for hip joint injections. Clin J Sport Med 2021;31:374-8.

25 Carubbi F, Bosch P, Machado PM, et al. Current practice of imaging-guided interventional procedures in rheumatic and musculoskeletal diseases: results of a multinational multidisciplinary survey. Front Med 2021;8.

26 Wambani IS, Korir GK, Tries MA, et al. Patient radiation exposure during general fluoroscopy examinations. J App/ Clin Med Phys 2014;15:262-70.

27 European Union Council. Council Directive 2013/59/Euratom of 5 December 2013 laying down basic safety standards for protection against the dangers arising from exposure to ionising radiation, and repealing directives 89/618/Euratom, 90/641/ Euratom, 96/29/Euratom, 97/43/Euratom a, 2014. Available: http://data.europa.eu/eli/ $\operatorname{dir} / 2013 / 59 / 0 j$

28 Cohen SP, Bicket MC, Kurihara C, et al. Fluoroscopically guided vs LandmarkGuided Sacroiliac joint injections: a randomized controlled study. Mayo Clin Proc 2019:94:628-42.

29 Hsu Y-C, Yang F-C, Hsu H-H, et al. Ultrasound-Guided corticosteroid injection in patients with carpal tunnel syndrome: efficacy of Intra-Epineurial injection. Ultraschall in Med 2018;39:334-42.

30 Chen P-C, Wang L-Y, Pong Y-P et al. Effectiveness of ultrasound-guided vs direct approach corticosteroid injections for carpal tunnel syndrome: a double-blind randomized controlled trial. J Rehabil Med 2018:50:200-8.

31 Roh YH, Hwangbo K, Gong HS, et al. Comparison of ultrasound-guided versus Landmark-Based corticosteroid injection for carpal tunnel syndrome: a prospective randomized trial. J Hand Surg Am 2019:44:304-10.

32 Pierre-Jerome C, Smitson RD, Shah RK, et al. Mri of the median nerve and median artery in the carpal tunnel: prevalence of their anatomical variations and clinical significance. Surg Radiol Anat 2010;32:315-22. 
33 Granata G, Caliandro P, Pazzaglia C, et al. Prevalence of bifid median nerve at wrist assessed through ultrasound. Neurol Sci 2011;32:615-8.

34 Choi CK, Lee HS, Kwon JY, et al. Clinical implications of real-time visualized ultrasound-guided injection for the treatment of ulnar neuropathy at the elbow: a pilot study. Ann Rehabil Med 2015;39:176-82.

35 Plessl D, Summey R, Joseph 0, et al. Feasibility of ultrasound-guided ulnar nerve injections at the cubital tunnel using a lateral-to-medial approach.J Musculoskelet Res 2014;17:1450002. X.

36 Hamscha UM, Tinhofer I, Heber S, et al. A reliable technique for ultrasound-guided perineural injection in ulnar neuropathy at the elbow. Muscle Nerve 2017;56:237-41.

37 Meng S, Tinhofer I, Grisold W, et al. Ultrasound-Guided perineural injection at Guyon's tunnel: an anatomic feasibility study. Ultrasound Med Biol 2015;41:2119-24.

38 Rampen AJJ, Wirtz PW, Tavy DL. Ultrasound-Guided steroid injection to treat mild ulnar neuropathy at the elbow. Muscle Nerve 2011;44:128-30.

39 Klauser AS, Abd Ellah MMH, Halpern EJ, et al. Meralgia paraesthetica: ultrasoundguided injection at multiple levels with 12-month follow-up. Eur Radiol 2016:26:764-70.

40 Mauri G, Gitto S, Pescatori LC, et al. Technical Feasibility of Electromagnetic US/CT Fusion Imaging and Virtual Navigation in the Guidance of Spine Biopsies. Ultraschall Med 2020;23. doi:10.1055/a-1194-4225. [Epub ahead of print: 12 Aug 2020].

41 Sartoris R, Orlandi D, Corazza A, et al. In vivo feasibility of real-time MR-US fusion imaging lumbar facet joint injections. J Ultrasound 2017;20:23-31.

42 Diffre C, Jousset C, Roux A-L, et al. Predictive factors for positive disco-vertebral biopsy culture in pyogenic vertebral osteomyelitis, and impact of fluoroscopic versus scanographic guidance. BMC Infect Dis 2020;20:512.

43 Rosenberg JM, Quint DJ, de Rosayro AM. Computerized tomographic localization of clinically-guided sacroiliac joint injections. Clin J Pain 2000;16:18-21.

44 Fouad AZ, Ayad AE, Tawfik KAW, et al. The success rate of Ultrasound-Guided Sacroiliac joint steroid injections in sacroiliitis: are we getting better? Pain Pract 2021;21:404-10.

45 Klauser AS, De Zordo T, Feuchtner GM, et al. Fusion of real-time us with CT images to guide sacroiliac joint injection in vitro and in vivo. Radiology 2010;256:547-53.

46 Cho C-H, Kim DH, Bae K-C, et al. Proper site of corticosteroid injection for the treatment of idiopathic frozen shoulder: results from a randomized trial. Joint Bone Spine 2016:83:324-9.
47 Khallaf SF, Hussein MI, El-Barbary AM, et al. Efficacy of ultrasonography-guided intra-articular steroid injection of the shoulder and excercising in patients with adhesive capsulitis: Glenohumeral versus subacromial approaches. The Egyptian Rheumatologist 2018;40:277-80.

48 Chang WH, Kim YW, Choi S, et al. Comparison of the therapeutic effects of intramuscular subscapularis and scapulothoracic bursa injections in patients with scapular pain: a randomized controlled trial. Rheumatol Int 2014;34:1203-9.

49 Park KD, Ahn JK, Lee S-C, et al. Comparison of ultrasound-guided intra-articular injections by long axis in plane approach on three different sites of the knee. Am J Phys Med Rehabil 2013:92:990-8.

50 Park Y, Lee SC, Nam H-S, et al. Comparison of sonographically guided intra-articular injections at 3 different sites of the knee. J Ultrasound Med 2011;30:1669-76.

51 Jang SH, Lee SC, Lee JH, et al. Comparison of ultrasound (US)-guided intra-articular injections by in-plain and out-of-plain on medial portal of the knee. Rheumatol Int 2013:33:1951-9.

52 Chang CY, Simeone FJ, Nelson SB, et al. Is biopsying the paravertebral soft tissue as effective as biopsying the disk or vertebral endplate? 10-year retrospective review of CT-guided biopsy of diskitis-osteomyelitis. AJR Am J Roentgenol 2015;205:123-9.

53 Mardani-Kivi M, Karimi-Mobarakeh M, Babaei Jandaghi A, et al. Intra-sheath versus extra-sheath ultrasound guided corticosteroid injection for trigger finger: a triple blinded randomized clinical trial. Phys Sportsmed 2018;46:93-7.

54 Shinomiya R, Sunagawa T, Nakashima Y, et al. Impact of corticosteroid injection site on the treatment success rate of trigger finger: a prospective study comparing ultrasound-guided true Intra-Sheath and true Extra-Sheath injections. Ultrasound Med Biol 2016;42:2203-8

55 Babaei-Ghazani A, Forogh B, Raissi GR, et al. Ultrasound-Guided corticosteroid injection in carpal tunnel syndrome: comparison between radial and ulnar approaches. J Pain Res 2020:13:1569-78.

56 Rayegani SM, Raeissadat SA, Ahmadi-Dastgerdi M, et al. Comparing The Efficacy Of Local Triamcinolone Injection In Carpal Tunnel Syndrome Using Three Different Approaches with or without Ultrasound Guidance]]\&gt. J Pain Res 2019;12:2951-8.

57 Babaei-Ghazani A, Nikbakht N, Forogh B, et al. Comparison between effectiveness of ultrasound-guided corticosteroid injection above versus below the median nerve in mild to moderate carpal tunnel syndrome. Am J Phys Med Rehabil 2018;97:407-13. 